\title{
La curva de Engel de los hogares en Medellín, Colombia 2012-2015
}

Engel Curves in Medellin's Households 2012 - 2015

\author{
Curvas de Engel nas Famílias de \\ Medellín, Colombia 2012-2015
}

Jorge Barrientos Marín* Efraín Arango Sánchez**

Fecha de envío: 8 de octubre de 2018

Fecha de aceptación: 27 de mayo de 2019

Cómo citar este artículo/ To reference this article / Comment citer cet article / Para citar este artigo:

Barrientos-Marín, J. \& Arango-Sánchez, E. (2019). La curva de Engel de los hogares en Medellín, Colombia 2012-2015. Apuntes del CENES, 38(68), 185 - 212. https://doi.org/10.19053/01203053.v38.n68.2019.8479

* Economista. PhD en Economía. Docente de la Universidad de Antioquia y la Universidad Autónoma Latinoamericana, Medellín, Colombia. jorge.barrientos@udea.edu.co.com @ https://orcid.org/0000-0001-5368-1928

** Economista. Magíster en Matemáticas. Docente de la Universidad Autónoma Latinoamericana. Medellín, Colombia. efrain.arango@unaula.edu.co https://orcid.org/0000-0003-2799-4984 


\section{Resumen}

Este artículo analiza y caracteriza el comportamiento del gasto y los patrones de consumo de los hogares de Medellín. El análisis se basa en la curva de Engel para hogares, la cual relaciona el gasto total con el gasto específico en ciertos bienes. Se recurrió a una metodología empírica basada en tres modelos: modelos semiparamétricos, para capturar no-linealidades, modelos de variables instrumentales y modelos Tobit. Se concluye que los bienes en alimentación, bebida y tabaco y vestido y calzado, responden a la ley de Engel, y dependiendo del segmento de gasto en el cual se ubiquen las familias, para algunas será un bien normal o inferior o de lujo.

Palabras clave: ley de Engel, gasto, ingreso, endogeneidad, variable instrumental, datos censurados, econometría.

Clasificación JEL: C01, C12, C33, D1, D12. 


\begin{abstract}
This paper analyzes and characterizes the consumer behavior and the consumption patterns in Medellin's households. We made the analysis based on the Engel curves for urban households, which relates the total expenditure to expenditure in specific goods. We perform three empirical models: semiparametric models to capture non-linearities, instrumental variables model because of the endogeneity and Tobit models due to censoring of the dependent variable. We concluded that food and beverage, tobacco and clothing and footwear, respond to the Engel Law, and depending on the expenditure segment in which the families are located, those goods could be normal, inferior or luxury.
\end{abstract}

Keywords: Engels' Law, expenditure, income, endogeneity, instrumental variable, data censoring, econometrics. 


\section{Resumo}

Este artigo analisa e caracteriza o comportamento do consumidor e os padrões de consumo nos domicílios de Medellin. Fizemos a análise com base nas curvas de Engel para as famílias urbanas, que relaciona a despesa total com a despesa em bens específicos. Realizamos três modelos empíricos: modelos semiparamétricos para capturar não-linearidades, modelo de variáveis instrumentais por causa dos modelos de endogeneidade e Tobit devido à censura da variável dependente. Concluímos que alimentos e bebidas, tabaco, roupas e calçados respondem à Lei de Engel e, dependendo do segmento de gastos em que as famílias estão localizadas, esses bens podem ser normais, inferiores ou de luxo.

Palavras-chave: Lei de Engel, gasto, renda, endogeneidade, variável instrumental, censura de dados, econometria. 


\section{INTRODUCCIÓN}

El análisis econométrico de las curvas de Engel ha sido tema de particular importancia entre los microeconomistas. Basta echar una mirada atrás en el tiempo sobre los artículos más influyentes publicados sobre el tema, por ejemplo: Working (1943), Leser (1963), Deaton y Muelbauer (1980a; 1980b), en los cuales desarrollaron estructuras paramétricas, hasta los más recientes estudios de Lyssiutou, Pashardes y Stengos (2002), Blundell, Browning y Crawford (2003), Barrientos (2009; 2011), los cuales realizan análisis estadísticos basados en modelos no-paramétricos o semiparamétricos que permiten más curvatura a las curvas estimadas. La mayoría de estos trabajos usan grandes encuestas de ingresos y gastos familiares que, desde luego, tienen problemas de endogenidad $y$ de censura en algunas de las variables dependientes.

La curva de Engel es un instrumento de análisis microeconómico que relaciona el ingreso de un agente con el nivel de demanda de determinados bienes
(Pindyck \& Rubinfeld (2016)). Sin embargo, empíricamente resulta de mayor interés la relación entre el gasto total y el gasto en determinados bienes específicos como alimentos, ropa, alquiler, etc. El análisis de las curvas de Engel tiene otras interesantes aplicaciones, que requiere una teoría de la integrablilidad más completa (es decir, cómo desde las demandas individuales es posible recuperar las funciones de utilidad), información estadística más depurada y técnicas de estimación un poco más sofisticadas que las propuestas aquí.

Las curvas de Engel permiten también, por ejemplo, llevar a cabo análisis de bienestar social, pero igualmente son importantes para hacer estudios de pobreza y distribución de ingresos. Incluso, una curva de Engel eventualmente permite saber si un bien es de lujo, inferior o normal, entre otras categorías de bienes. Algo que suele ser de interés no solo para los mircoeconomistas, sino también para los hacedores de política pública. 
El objetivo de este trabajo es estimar la relación que existe entre el gasto total de los hogares en Medellín durante el periodo 2012-2015 y la participación del gasto en diferentes rubros como alimentación, tabaco y licor y vestido y calzado como porcentaje del gasto total. Adicionalmente, se estima la media condicional, controlando por las características del hogar y del jefe de hogar, como por ejemplo sexo, edad y estrato socioeconómico y número de personas por hogar. La hipótesis de trabajo es que los datos correspondientes a zonas urbanas, como es el caso de Medellín, respaldan la teoría microeconómica detrás de las curvas de Engel.

La información utilizada en este trabajo resulta de la concatenación de cuatro bases de datos: las encuestas de calidad de vida de Medellín de los años 2012 al año 2015. Se descarta la información de 2011 hacía atrás por problemas de datos faltantes en muchas de las variables de interés, incluido el gasto de los hogares. Como los hogares encuestados cada año no son los mismos, evidentemente no hay un panel de datos Baltagui (2005). De modo que cada hogar se analiza como una observación independiente, que de hecho lo es. Esta encuesta es financiada por la Alcaldía de Medellín y llevada cabo por el Centro de Estudios de Opinión de la Universidad de Antioquia.

La estrategia empírica desarrollada en esta investigación está basada en tres modelos diferentes: un modelo no-paramétrico para capturar no-linealidades, regresiones por mínimos cuadrados en dos etapas (MC2E) para corregir la endogeneidad por simultaneidad y regresiones tipo Tobit para corregir el sesgo debido a la censura de la variable dependiente, en particular en el gasto en alimentación. Cada uno de los resultados muestra una consistencia con lo que predice la teoría económica del consumidor y la ley de Engel.

Contribuciones de este trabajo: i) investigamos el comportamiento, el patrón de consumo de los agentes estimando un sistema de curvas de Engel en donde se incluyen en las regresiones algunas características del jefe de hogar que por su naturaleza entran de forma lineal a ser, la mayoría de ellas, variables categóricas; ii) se ajustan las estimaciones por endogeneidad usando el método de Sperlich (2005), tomando el ingreso corriente de la familia y otras variables explicativas exógenas como instrumentos para el gasto total. Nótese que mientras las decisiones de los agentes sobre gasto son internas al hogar, el ingreso familiar es un factor determinado externamente; más aún, este se determina por el mercado laboral, lo cual lo convierte en un excelente instrumento para el gasto total; iii) se estiman modelos Tobit. Este sería, hasta donde tenemos conocimiento, el primer artículo en la literatura nacional que trata el problema del sesgo ocasionado por la censura de la variable dependiente. 
Los artículos relacionados con este trabajo son los de López y Gómez (1976), Banks, Blundell y Lewbel (1997), Bhalotra y Attfield (1998), Blundell, Duncan y Pendakur (1998), Lyssiotou et al. (2002), Blundell et al. (2003), Ramírez, Cortes y Gallego (2002), Lasso (2003), Barrientos (2009, 2011), Moral-Arcte, Rodriguez-Poo y Sperlich (2013) y sus referencias. Con respecto al caso colombiano, vale la pena señalar que no hay muchos artículos sobre el comportamiento del consumidor en Colombia.

Este trabajo se divide en cuatro secciones, además de esta introducción. En la primera sección se presenta el marco conceptual; en la segunda se explica la estrategia empírica; en la tercera sección se presentan los resultados; en la sección cuarta se exponen algunas conclusiones, y en último término se incluyen anexos con los resultados completos para el año 2015. Para los demás años, junto con los códigos en Stata, están disponibles a solicitud de los lectores.

\section{MARCO CONCEPTUAL}

En la teoría microeconómica clásica los individuos maximizan la utilidad bajo una restricción de presupuesto $-\mathrm{O}$ nivel de riqueza determinado-, el cual es exógeno a las decisiones de consumo:

$$
\begin{aligned}
& \max _{\mathbf{x} \in X} u(\mathbf{x}) \\
& \mathbf{p x} \leq m
\end{aligned}
$$

Donde $u: X \rightarrow \mathbb{R}$, es una función continua y derivable, $X \subseteq \mathbb{R}^{n}$ es el conjunto de consumo que es compacto y convexo, $\mathbf{p}=\left(p_{1}, \ldots, p_{n}\right)^{\prime} \mathrm{y} \mathbf{x}=\left(x_{1}, \ldots, x_{n}\right)^{\prime}$ son los vectores de precios y de bienes y la renta está dada por $m \in \mathbb{R}$. La solución de este tipo de problemas lleva a determinar las funciones de demanda que explican de qué manera afectan las variables socioeconómicas involucradas las decisiones de consumo. Típicamente, las demandas óptimas son funciones de los precios y de la renta.

Este trabajo se enfoca específicamente en el estudio de las curvas de Engel como resultado del análisis de las curvas de renta-consumo, las cuales muestran las combinaciones de cestas de bienes maximizadoras de utilidad que corresponden a todos los niveles de renta. Así, para cada nivel posible de renta $m \in \mathbb{R}$ del individuo, existen nuevos puntos de óptimo en el problema (1) y naturalmente nuevos puntos de consumo de bienes; por ejemplo nuevos niveles de demanda del par de bienes $\left(x_{k, m}^{*}, x_{j, m}^{*}\right)$ a distintos niveles de renta $m$ (fijos los precios $p_{k}$ y $p_{j}$ ), así las demandas óptimas de bienes se vuelven una función de la renta (que es igual al gasto en el equilibrio parcial), constituyendo así la curva de Engel:

$$
x_{k, m}^{*}=g(m)
$$

En términos generales, las curvas de Engel muestran la relación que existe entre la renta de los individuos y las cantidades demandadas de determinado 
bien o conjunto de bienes. Del análisis de la curva de Engel para cada bien es posible determinar si los bienes son normales, inferiores o si estos muestran algún grado de sensibilidad a las variaciones de los niveles de renta.

Sin embargo, a diferencia del modelo teórico en la ecuación (2), la especificación empírica propuesta implica una redefinición de la curva de Engel en términos del gasto total, GT, y otras variables socioeconómicas, denotadas por $Z$, que influencian el gasto en bienes específicos. Así, se tiene que la proporción como porcentaje del gasto total en el bien $k$, de un hogar típico $i$, está dado por,

$$
\frac{p_{k} x_{i, k, G}^{*}}{G T_{i}}=w_{i, k, G}=f\left(G T_{i}, Z_{i}\right)
$$

Es importante notar que la ecuación (3) da lugar a un modelo de regresión cuya especificación depende crucialmente de los supuestos sobre $f(\cdot)$, la estructura de datos subyacente y la distribución de probabilidades de las perturbaciones asociadas a todo modelo de regresión, así como también la censura de la variable dependiente, $w_{i k}$, y de la correlación entre el gasto total de los hogares y las perturbaciones del modelo de regresión, esto es de la endogeneidad del gasto total.

\section{METODOLOGÍA}

Un problema técnico que se mencionó anteriormente es el de la endogeneidad de la variable gasto total, el cual es muy frecuente en este tipo de estimaciones; en particular, porque el gasto total y el gasto en algún rubro (alimentos, ropa, salud, etc.) están conjuntamente determinados, lo que produce endogeneidad por doble causalidad. Vale la pena aclarar que la endogeneidad puede también tener origen en la errónea medición de las variables de interés. En cualquier caso, la solución al problema de estimación, que causa estimadora inconsistente, está en el método de momentos generalizado (GMM) y su caso particular: variables instrumentales a través de una estimación por MC2E.

Existen diversos métodos para aplicar variables instrumentales. Desde el punto de vista paramétrico se suele utilizar la técnica de mínimos cuadrados en dos etapas (2SLS), basados en el método GMM. Newey y Powell (2003) desarrollaron un método no paramétrico en dos etapas; sin embargo, este es complicado de implementar porque requiere la elección de una base (vectorial) expandible en una primera etapa. Existe otra aproximación rápida y eficiente debida a Sperlich (2005) y conocida como regresores construidos en dos etapas. Barrientos (2009) usando la Encuesta de Calidad de Vida del DANE para Bogotá, aplica este método en un análisis de consumo de alimentos, ropa y transporte con resultados satisfactorios.

El trabajo empírico en este artículo comienza con un diagnóstico sobre la presencia de no-linealidades en la relación entre la proporción del gasto total 
dedicada a un bien específico, como el de la ecuación [3]. Sea $w_{k i}$ la proporción del gasto total en el bien $k$ del hogar $i$, $\mathrm{y}$ sea $\mathbf{x}_{i}$ un vector $k \times 1$ de variables explicativas (número de personas por hogar, el gasto total del hogar (GT), estrato socioeconómico, edad y género del jefe de hogar). La detección de tal no linealidad se puede llevar a cabo haciendo una regresión no-paramétrica unidimensional del tipo:

$$
w_{k i}=m\left(x_{G T i}\right)+\varepsilon_{i} \quad i=1, \ldots, N
$$

Donde $x_{G T i}$ es el gasto total (GT) del hogar $i$. Un estimador de $m(\cdot)$ puede ejecutarse usando un estimador núcleo (Nadaraya-Watson, 1964).

Un método considerado, con fines de comparación, es obtener una estimación de los parámetros de interés en una regresión lineal de $w_{k i}$ sobre el vector $\mathbf{x}_{i}$,

$$
w_{k i}=\mathbf{x}_{i}^{\prime} \beta+\varepsilon_{i}, \quad E\left(\mathbf{x}_{i}^{\prime} \varepsilon_{i}\right)=0
$$

El método habitual es mínimos cuadrados ordinarios (MCO). Es importante notar que entre el vector $\mathbf{x}_{i}^{\prime}$ se encuentra, además del gasto total, el cuadrado del gasto total, en particular si la regresión en [4] sugiere no-linealidad de la relación entre $w_{k i}$ y el GT.

El segundo método considerado es un método generalizado de momentos (GMM), específicamente mínimos cuadrados en dos etapas (MC2E), como se indicó, debido a la endogeneidad por simultaneidad del GT, esto es,

$$
\begin{gathered}
w_{k i}=\mathbf{x}_{i}^{\prime} \beta+\varepsilon_{i}, \\
E\left(\mathbf{x}_{i}^{\prime} \varepsilon_{i}\right) \neq 0 \\
\text { y } E\left(\mathbf{z}_{i}^{\prime} \varepsilon_{i}\right)=0
\end{gathered}
$$

Donde $\mathbf{z}_{i}^{\prime}$ es una matriz de instrumentos, que en el cálculo de la curva de Engel suele ser alguna medida del ingreso o, si se dispone, el ingreso del hogar más las variables exógenas en el modelo. Es importante mencionar que el ingreso del hogar está generalmente medido con error. Sea $y_{i}$ el ingreso del hogar $i$. Como el ingreso está medido con error, esto es, lo observado suele ser una medición imperfecta del ingreso, entonces lo que realmente se observa es la variable medida con error,

$$
y_{i}^{*}=y_{i}+e_{i}
$$

Donde $e_{i}$ es el error de medida tal que $E\left(e_{i}\right)=0$ para todo $i$. Claramente, asumiendo que $E\left(e_{i} \varepsilon_{i}\right)=0 \forall i$, lo que no es un supuesto muy fuerte, habida cuenta de que el error de la medida en [7] no debe estar correlacionado con el error de la regresión en [6], pues su origen es muy diferente y permite que el ingreso sea un instrumento válido para estimar la ecuación [6].

El tercer método considerado es un procedimiento Tobit, pues el gasto reportado por algunos hogares en algunos rubros es cero (0), lo que claramente es una anomalía porque gasto cero (0) 
anual en bienes como alimentación, bebida/tabaco y ropa/calzado, suele ser un indicativo de que la variable dependiente tiene censura. El modelo censurado viene dado por la siguiente especificación empírica,

$$
\begin{aligned}
& w_{i}^{*}=\mathbf{x}_{i}^{\prime} \beta+\varepsilon_{i} \\
& w_{i}=0 \quad \text { si } w_{i}^{*} \leq 0 \\
& w_{i}=w_{i}^{*} \text { si } w_{i}^{*}>0
\end{aligned}
$$

La estimación de los parámetros de interés en la ecuación [8] es por máxima verosimilitud, pues el modelo de regresión subyacente es no lineal, de hecho la esperanza condicional por estimar es,

$$
E\left(w_{i}^{*} \mid w_{i}^{*}>0\right)=\mathbf{x}_{i}^{\prime} \beta+\sigma \lambda_{i}
$$

Donde $\sigma \lambda_{i}$ es el sesgo producido por la censura en la variable dependiente y $\lambda_{i}$ se conoce como el inverso de la razón de Mills.

\section{RESULTADOS}

Los datos utilizados en este proyecto de investigación se obtuvieron de la Encuesta de Calidad de Vida de Medellín 2012-2015. En principio, los datos disponibles eran para el período 20082013; sin embargo, debido a la falta de información en muchas variables, descartamos el uso de la información desde 2011 hacía atrás. Vale decir que después de un esfuerzo importante se pudieron conseguir y concatenar los años restantes, de modo que finalmente el período analizado es 2012-2015. Es importante anotar que las variables independientes son binarias - excepto el número de personas por hogar y la edad del jefe de hogar, que son continuas-; así por ejemplo, el $\operatorname{Sexo}_{i}$ del jefe del hogar $i$ es una variable binaria que toma el valor $=1 \mathrm{si}$ el jefe de hogar es hombre y toma el valor de 0 en otro caso, al igual que cada estrato. Las variables dependientes son continuas.

La Tabla 1 expone las estadísticas descriptivas de los hogares en la muestra. Se tiene una muestra de alrededor de 48 000 hogares; el número de personas por hogar es cercano a los 3.5, la edad reportada por los jefes de hogar es de 51.7 años. El $53 \%$ de los jefes de hogar son hombres, el resto son mujeres, un dato no menos importante y que refuerza la tendencia de las mujeres en años recientes a hacerse cargo del hogar. El $35 \%$ de los hogares reporta ser de estrato 2 , mientras que el $29 \%$ reporta ser de estrato 3 y el $5 \%$, de estrato 6 . Como es de esperarse, y coherente con la Figura 1 , los ingresos promedios reportados son inferiores a los gastos, además, como también es de esperarse, el gasto en alimentación es con diferencia uno de los rubros que ocupa una proporción mayor del gasto total (y del ingreso, por supuesto). 
Tabla 1. Estadísticas descriptivas, 2012-2015

\begin{tabular}{c|ccccc}
\hline Variables & N. ${ }^{\circ}$ Obs. & Media & Des. Est. & Mín & Máx \\
\hline N. ${ }^{\circ}$ Personas x Hogar & 48.189 & 3,54 & 1,67 & 1 & 20 \\
Sexo & 48.189 & 0,53 & ----- & 0 & 1 \\
Edad & 48.189 & 51,75 & 15,41 & 16 & 85 \\
Estrato 1 & 48.189 & 0,12 & ----- & 0 & 1 \\
Estrato 2 & 48.189 & 0,35 & ----- & 0 & 1 \\
Estrato 3 & 48.189 & 0,29 & ----- & 0 & 1 \\
Estrato 4 & 48.189 & 0,11 & ----- & 0 & 1 \\
Estrato 5 & 48.189 & 0,08 & ------ & 0 & 1 \\
Estrato 6 & 48.189 & 0,04 & ----- & 0 & 1 \\
Ingreso total & 47.028 & 12.900 .000 & 12.600 .000 & 1200 & 121.000 .000 .000 \\
Gasto total & 48.921 & $14.900 .000,00$ & $20.900 .000,00$ & $18.000,00$ & $1.200 .000 .000,00$ \\
Gasto en alimentación & 48.921 & $5.341 .239,00$ & $3.800 .257,00$ & 0 & $120.000 .000,00$ \\
Gasto alcohol y tabaco & 47.028 & 179.334 & 648520 & 0 & 3.600 .000 \\
Gasto en vestido y & 47.028 & 805.542 & 1025068 & 0 & 3.000 .000 \\
calzado & & & & & \\
\hline
\end{tabular}

Fuente: cálculos propios con base en ECVMED 2012-2015.

La Figura 1 muestra la evolución del los hogares se ha deteriorado en los úlgasto promedio anual de las familias de timos años, como consecuencia natural Medellín, en el período 2012-2015. Se de la desaceleración del ingreso. encontró evidencia de que el gasto de

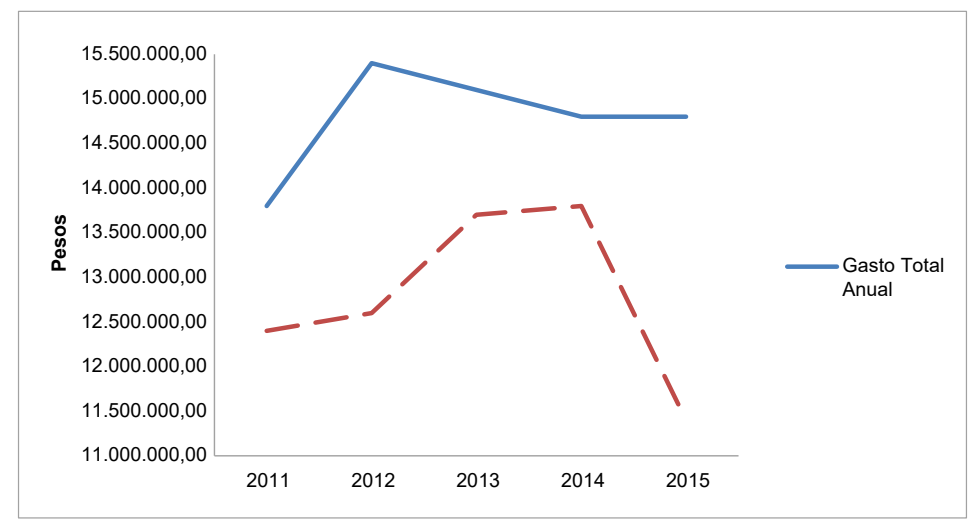

Figura 1. Ingreso y gasto promedio de Medellín.

Fuente: cálculos propios con base en ECVMED 2012-2015. 
Se muestran los resultados de las estimaciones econométricas para los modelos no-paramétricos y paramétricos. Primero, los resultados correspondientes al periodo 2012-2015 y, posteriormente, año a año. Puesto que los resultados año a año no varían mucho con respecto al periodo completo, en el anexo al final del documento se encuentran las estimaciones para los años 2015 .

Estimaciones para el período 20122015: estimación no-paramétrica

La estimación de la ecuación 4 se lleva cabo usando estimadores tipo núcleo, el bien conocido estimador Nadaraya-Watson (1964), dado por:

$$
\widehat{m}(x)=\frac{\sum_{i=1}^{N} K_{h}\left(X_{i}-x\right) Y_{i}}{\sum_{i=1}^{N} K_{h}\left(X_{i}-x\right)}
$$

Donde $K_{h}(\cdot)$ es la función kernel y $h>0$ es el ancho de banda o bandwidth. Es importante notar que la estimación no-paramétrica lo que estima es una función "continua", de modo que lo habitual es presentar un gráfico de los valores ajustados de la variable dependiente contra la variable independiente en este caso.

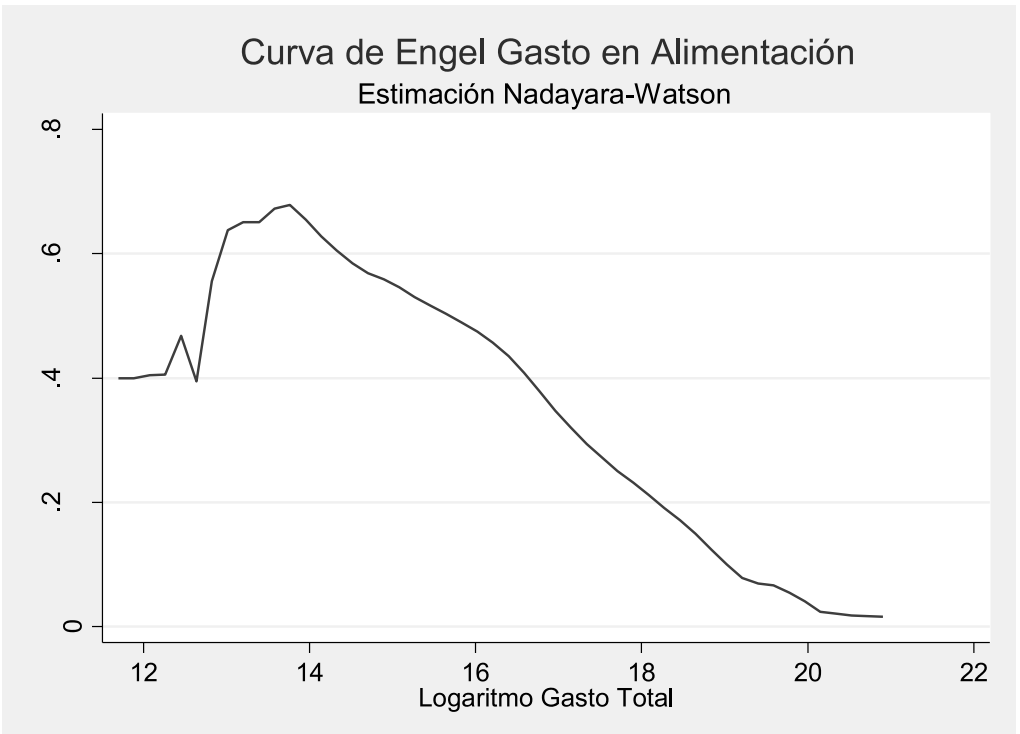

Figura 2. Curva de Engel: gasto en alimentación 


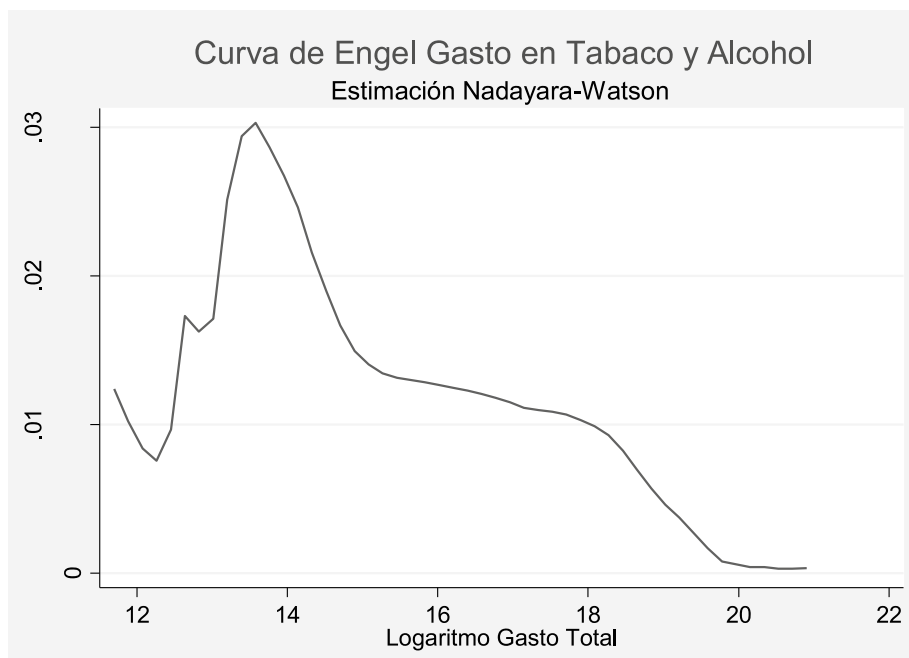

Figura 3. Curva de Engel: gasto en tabaco y alcohol

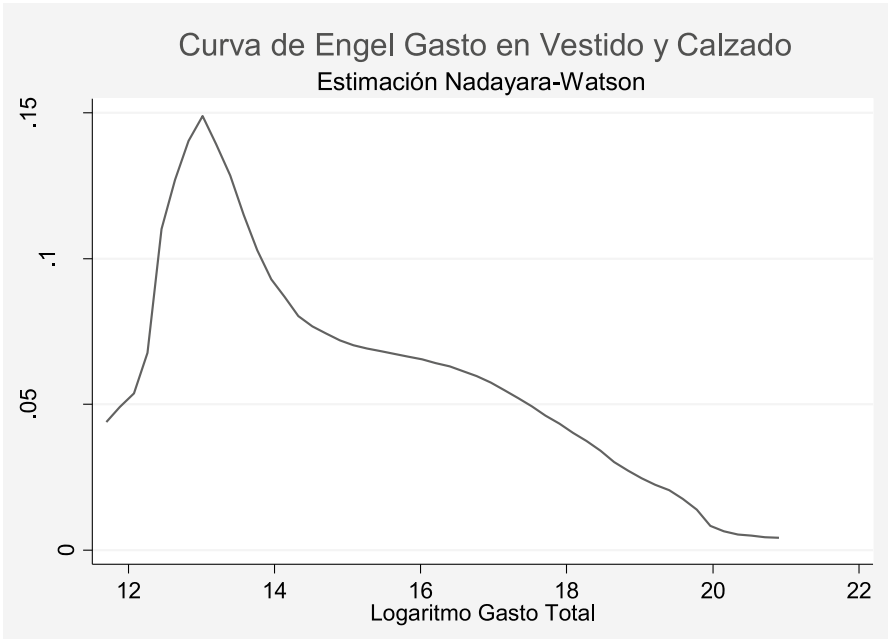

Figura 4. Curva de Engel: gasto en vestido y calzado

Las figuras 2 al 4 muestran los resultados de las estimaciones no-paramétricas (ecuación 4). Nótese que la función $m(\cdot)$ es unidimensional, pues el interés es encontrar evidencia de no-linealidades en la relación entre las participaciones de los diferentes rubros de gasto $\left(w_{k}\right)$ en el gasto total del hogar. Efectivamente, las figuras 2 a 4 muestran que es necesario introducir más curvatura a la regresión multidimensional, y que efectivamente el gasto total debe entrar en el modelo 
de regresión no solo de manera lineal sino cuadrática. De modo que en el modelo base (ecuaciones 5, 6 y 8) la parte determinística $\mathbf{x}_{i}^{\prime} \beta$ puede ser escrita como,

$$
\begin{aligned}
& \mathbf{x}_{i}^{\prime} \beta=\beta_{0}+\beta_{1} \ln \left(G T_{i}\right) \\
& +\beta_{2}\left(\ln \left(G T_{i}\right)\right)^{2}+\mathbf{x}_{i}^{\prime *} \beta^{*}
\end{aligned}
$$

Por tanto, el modelo lineal en parámetros base está dado por:

$$
\begin{gathered}
w_{k i}=\mathbf{x}_{i}^{\prime} \beta+\varepsilon_{i}=\beta_{0}+\beta_{1} \ln \left(G T_{i}\right) \\
+\beta_{2}\left(\ln \left(G T_{i}\right)\right)^{2}+\mathbf{x}_{i}^{\prime *} \beta^{*}+\varepsilon_{i}
\end{gathered}
$$

Donde $\mathbf{x}_{i}^{\prime *}$ es el resto de variables explicativas (edad, sexo, estrato, N. ${ }^{o}$ Pers. $\mathrm{x}$ hogar, etc.). La ecuación [1] es estimada por OLS, por variables instrumentales y por regresión censurada.

\section{Estimación paramétricas: gasto en alimentación}

Las figuras 5 al 7 muestran la estimación de la curva de Engel paramétrica. La Tabla 2 indica los resultados de los parámetros estimados para las distintas regresiones, cuya variable dependiente es el gasto en alimentación. La columna 1 muestra la estimación OLS; la columna 2, la estimación censurada, y la columna 3 , la estimación por variables instrumentales. Cada parámetro va acompañado de la $t$-student entre paréntesis (con lo que se calcula el error estándar) para analizar la significancia individual de las variables explicativas.
De acuerdo con las estimaciones que se llevaron a cabo, y teniendo en cuenta al gasto total como una medida de la renta, se observa que para aquellos hogares que poseen unos niveles de renta menores, en las dos primeras estimaciones la pendiente de la curva de Engel es negativa, lo cual permite conjeturar que, en general, todos los hogares que fueron tenidos en cuenta dentro de la muestra considerarían al gasto en alimentación como un bien inferior, ya que a mayores niveles de ingresos, la proporción del gasto total en alimentación disminuye.

Esta conclusión podría ajustarse en cierta medida a los hogares con mayores niveles de ingresos, pero rara vez podría suceder lo mismo con los hogares de menores ingresos, los cuales destinan un porcentaje muy alto de sus ingresos al tema de alimentación. Estos resultados iniciales pueden atribuirse a la censura de los datos y la existencia de endogeneidad.

En el caso de variables instrumentales, la curva que se obtiene es parabólica, más acorde con la teoría microeconómica y con la evidencia empírica reportada en la mayoría de autores ya citados. Esto significa que $\mathrm{MC} 2 \mathrm{E}$ provee resultados más consistentes que MCO. La forma de la curva permite establecer que para el caso de los hogares con menores ingresos las rectas tangentes trazadas a esta curva tienen pendiente positiva, lo que nos muestra una relación directa entre el gasto total y el gasto en alimentación. Por lo tanto, para estos hogares el gasto 
en alimentación se considera un bien normal, pues a medida que aumentan los ingresos, aumenta el porcentaje del mismo que se destina a este rubro. Además, para los hogares de mayores ingresos, el porcentaje destinado a alimentación disminuye, lo cual indica que para estos hogares el gasto en alimentación puede considerarse como un bien inferior.

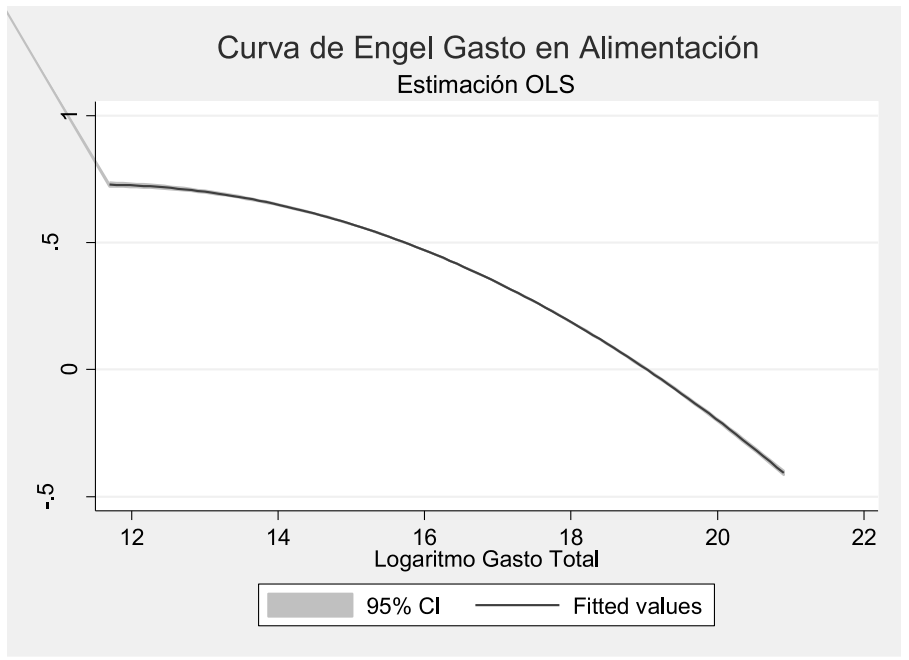

Figura 5. Curva de Engel: gasto en alimentación OLS

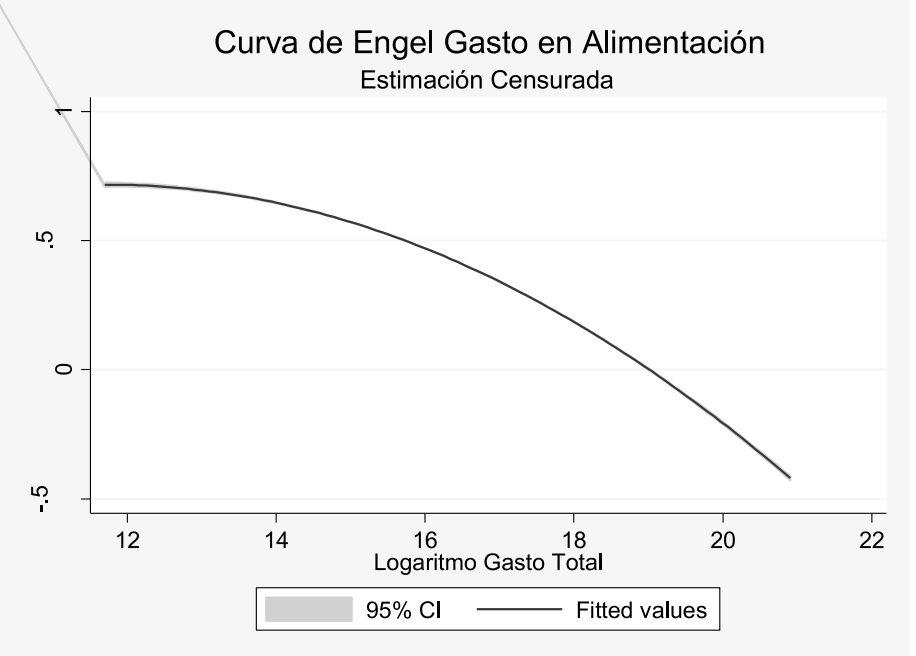

Figura 6. Curva de Engel: gasto en alimentación estimación censurada 


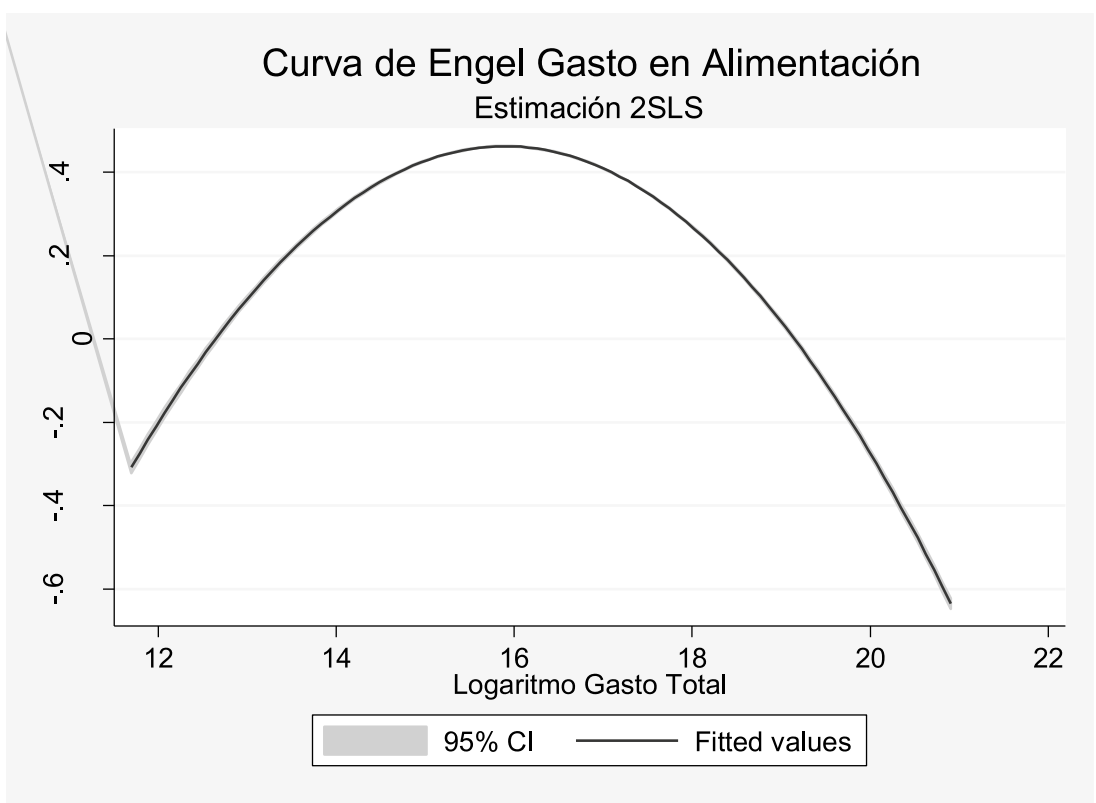

Figura 7. Curva de Engel: gasto en alimentación 2SLS

\section{Gasto en bebida y tabaco}

Las figuras 8 a 10 y la Tabla 3 muestran los resultados de las regresiones OLS, Tobit y variables instrumentales para el caso del gasto en bebida y tabaco. Nótese que la estimación OLS es opuesta a la estimación censurada y a la estimación por variables instrumentales, puesto que efectivamente los datos son censurados y existe endogeneidad del gasto total. Claramente, la estimación OLS es menos confiable, en el sentido de no capturar la media condicional de manera adecuada, dado que no considera el problema de especificación introducido por la ausencia de exogeneidad y por la censura en la variable dependiente.
De acuerdo con la estimación de mínimos cuadrados ordinarios (OLS), la relación entre el gasto total y el gasto en tabaco y alcohol es inversamente proporcional en la mayoría del intervalo definido; por lo tanto, para la mayoría de estos hogares el gasto en alcohol y tabaco es considerado un bien inferior. Por otro lado, para unos pocos hogares de ingresos muy altos, el gasto en alcohol y tabaco sigue comportamientos cercanos a un bien normal, debido al cambio en la pendiente de la curva estimada.

En el caso de la estimación Tobit, la curva obtenida da cuenta de una relación directa entre el gasto en alcohol y tabaco y el gasto total. La curva es estricta- 
mente creciente en todo el intervalo de evaluación. Es decir, tanto los hogares con menores ingresos, como aquellos con ingresos más altos consideran al gasto en tabaco y alcohol como un bien normal.

Tabla 2. Estimación curva de Engel: gasto en alimentación, 2012-2015

\begin{tabular}{|c|c|c|c|}
\hline Regresión & OLS & Tobit & $\begin{array}{c}\text { Variables } \\
\text { instrumentales }\end{array}$ \\
\hline Variable dependiente (Wa) & Coeficiente & Coeficiente & Coeficiente \\
\hline \multirow[t]{2}{*}{ N. ${ }^{\circ}$ personas por hogar } & 0,0180 & 0,0179 & 0,0045 \\
\hline & $(35,90)$ & $(39,65)$ & $(7 ., 96)$ \\
\hline \multirow[t]{2}{*}{ Sexo } & 0,0199 & 0,0198 & 0,0005 \\
\hline & $(13,57)$ & $(13,60)$ & $(0,31)$ \\
\hline \multirow[t]{2}{*}{ Ln(edad) } & 0,0565 & 0,0566 & 0,0691 \\
\hline & $(25,00)$ & $(25,08)$ & $(26,56)$ \\
\hline \multirow[t]{2}{*}{$\operatorname{Ln}(G T * G T)$} & $-0,0132$ & $-0,0138$ & $-0,0378$ \\
\hline & $(9,78)$ & $(16,76)$ & $(11, .65)$ \\
\hline \multirow[t]{2}{*}{$\operatorname{Ln}(\mathrm{GT})$} & 0,3082 & 0,3269 & 1,2604 \\
\hline & $(6,87)$ & $(12,13)$ & $(11,84)$ \\
\hline \multirow[t]{2}{*}{ Estrato 2} & $-0,0232$ & $-0,0233$ & $-0,0640$ \\
\hline & $(8,81)$ & $(9,70)$ & $(20,56)$ \\
\hline \multirow[t]{2}{*}{ Estrato 3} & $-0,0413$ & $-0,0415$ & $-0,1430$ \\
\hline & $(14,27)$ & $(15,89)$ & $(39,42)$ \\
\hline \multirow[t]{2}{*}{ Estrato 4} & $-0,0419$ & $-0,0425$ & $-0,2050$ \\
\hline & $(11,24)$ & $(12,69)$ & $(44,85)$ \\
\hline \multirow[t]{2}{*}{ Estrato 5} & $-0,0350$ & $-0,0356$ & $-0,2388$ \\
\hline & $(8,34)$ & $(9,32)$ & $(45,53)$ \\
\hline \multirow[t]{2}{*}{ Estrato 6} & $-0,0131$ & $-0,0132$ & $-0,2665$ \\
\hline & $(2,54)$ & $(2,66)$ & $(36,48)$ \\
\hline \multirow[t]{2}{*}{ Constante } & $-1,3282$ & $-1,4802$ & $-10,2091$ \\
\hline & $(3,59)$ & $(6,72)$ & $(11,71)$ \\
\hline Número de observaciones & 48.189 & 48.189 & 48.189 \\
\hline Coeficiente de determinación & 0,2786 & $* * *$ & 0,0811 \\
\hline
\end{tabular}




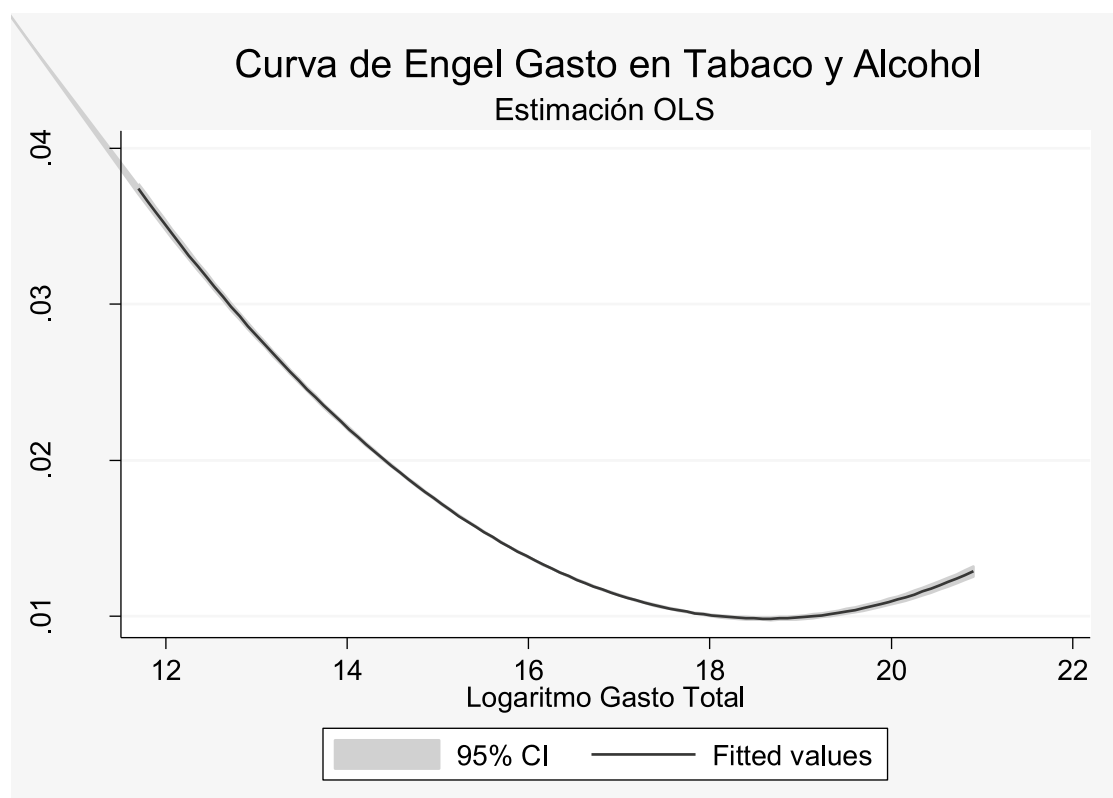

Figura 8. Curva de Engel: gasto en tabaco OLS

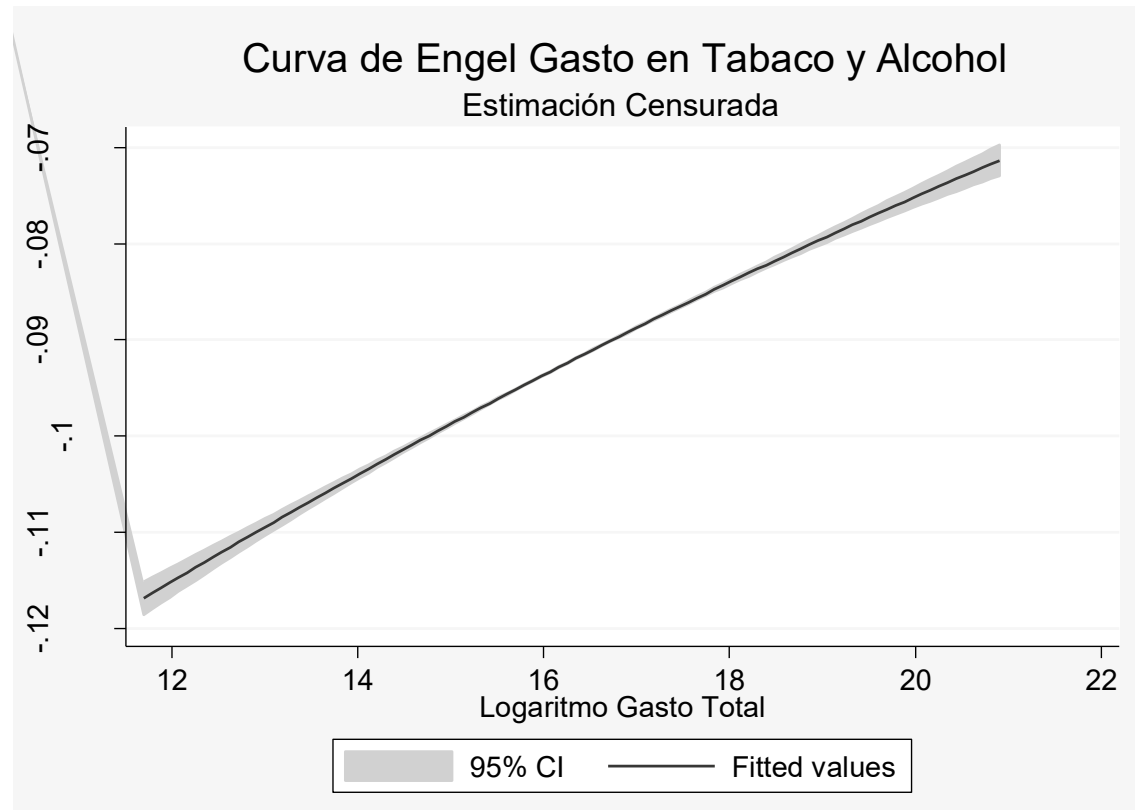


Figura 9. Curva de Engel: gasto en tabaco y alcohol censurada

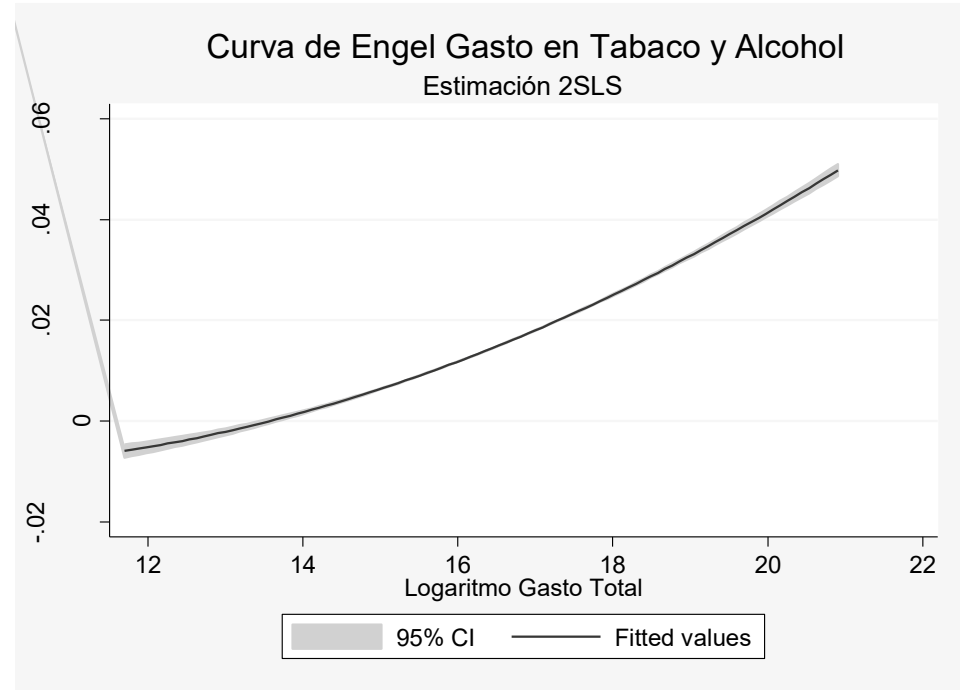

Figura 10. Gasto en tabaco y alcohol 2SLS

En el caso de variables instrumentales, tos, la relación es directamente proporla concavidad de la curva varía con cional, a mayores ingresos, mayor es el respecto al modelo de Tobit, pero tanto gasto en tabaco y alcohol, esto implica para los hogares de menores ingresos normalidad del rubro con respecto a los como para aquellos de ingresos más al- ingresos.

Tabla 3. Estimación curva de Engel: gasto en bebida y tabaco, 2012-2015

\begin{tabular}{c|ccc}
\hline Regresión & OLS & Tobit & $\begin{array}{c}\text { Variables } \\
\text { instrumentales }\end{array}$ \\
\hline Variable dependiente $(W b t)$ & Coeficiente & Coeficiente & Coeficiente \\
\hline N. ${ }^{\circ}$ Personas por hogar & $-0,0003$ & 0,0000 & $-0,0016$ \\
& $(2,28)$ & $(0,06)$ & $(10,95)$ \\
\hline Sexo & 0,0022 & 0,0089 & 0,0006 \\
& $(6,28)$ & $(6,08)$ & $(1,41)$ \\
\hline Ln(edad) & 0,0000 & $-0,0008$ & 0,0006 \\
& $(0,05)$ & $(0,38)$ & $(0,97)$ \\
\hline $\operatorname{Ln}\left(\mathrm{GT}^{*} \mathrm{GT}\right)$ & 0,0004 & 0,0003 & 0,0013 \\
& $(1,45)$ & $(0,30)$ & $(3,12)$ \\
\hline
\end{tabular}




\section{Continuación Tabla 3}

\begin{tabular}{|c|c|c|c|}
\hline $\operatorname{Ln}(\mathrm{GT})$ & $\begin{array}{c}-0,0147 \\
(1,66)\end{array}$ & $\begin{array}{l}0,0021 \\
(0,08)\end{array}$ & $\begin{array}{c}-0,0300 \\
(2,18)\end{array}$ \\
\hline Estrato 2 & $\begin{array}{c}-0,0030 \\
(0,57)\end{array}$ & $\begin{array}{c}-0,0038 \\
(1,61)\end{array}$ & $\begin{array}{r}-0,0037 \\
(4,95)\end{array}$ \\
\hline Estrato 3 & $\begin{array}{c}-0,0018 \\
(2,71) \\
\end{array}$ & $\begin{array}{c}-0,0140 \\
(5,35)\end{array}$ & $\begin{array}{l}-0,0113 \\
(13,45)\end{array}$ \\
\hline Estrato 4 & $\begin{array}{c}-0,0017 \\
(1,94)\end{array}$ & $\begin{array}{c}-0,0193 \\
(5,69)\end{array}$ & $\begin{array}{r}-0,0183 \\
(16,51) \\
\end{array}$ \\
\hline Estrato 5 & $\begin{array}{c}-0,0016 \\
(1,60)\end{array}$ & $\begin{array}{c}-0,0247 \\
(6,37)\end{array}$ & $\begin{array}{r}-0,0241 \\
(17,67)\end{array}$ \\
\hline Estrato 6 & $\begin{array}{c}-0,0001 \\
(0,04)\end{array}$ & $\begin{array}{c}-0,0182 \\
(3,70)\end{array}$ & $\begin{array}{l}-0,0313 \\
(16,95)\end{array}$ \\
\hline Constante & $\begin{array}{l}0,1509 \\
(2,03) \\
\end{array}$ & $\begin{array}{c}-0,0186 \\
(0,83)\end{array}$ & $\begin{array}{r}0,1593 \\
(1,44) \\
\end{array}$ \\
\hline Número de observaciones & 59.488 & 48.074 & 48.074 \\
\hline Coeficiente de determinación & 0,0028 & $* * *$ & 0.025 \\
\hline
\end{tabular}

Gasto en vestido y calzado

Las figuras 8 a 10 y la Tabla 4 muestran los resultados de las regresiones OLS, Tobit y variables instrumentales para el caso del gasto en vestido y calzado. En el caso correspondiente al gasto en vestido y calzado, las estimaciones iniciales por mínimos cuadrados ordinarios (OLS) y Tobit indican que los hogares que conforman el intervalo de análisis, es decir, tanto los que poseen unos ingresos bajos como aquellos de ingresos más altos, consideran que el gasto en vestido y calzado tiene un comportamiento que se asemeja al de un bien inferior, a medida que el gasto total se incrementa, la proporción que se destina a vestido y calzado disminuye. Aunque en ambos casos, las pendientes de las curvas son negativas, en el caso Tobit la curvatura es mayor, lo que se traduce en un mayor grado de inferioridad.

En el caso de variables instrumentales, la curva que se obtiene es parabólica; la forma de esta curva nos permite establecer que para el caso de los hogares con menores ingresos las rectas tangentes trazadas a esta curva tienen pendiente positiva, lo que nos señala una relación directa entre el gasto total y el gasto en vestido y calzado. Por lo tanto, para estos hogares el gasto en vestido y calzado 
se considera un bien normal, a medida que aumentan los ingresos, aumenta el porcentaje del mismo que se destina a este rubro. Además, para los hogares de mayores ingresos, el porcentaje destinado a vestido y calzado disminuye, lo cual indica que para estos hogares el gasto en alimentación puede considerar- se como un bien inferior. Otra forma de analizar estos fenómenos tiene que ver con el hecho de que cuando los hogares de alguna forma tienen cubierto alguno de estos rubros, los mayores niveles de ingresos pueden ser destinados a otros menesteres.

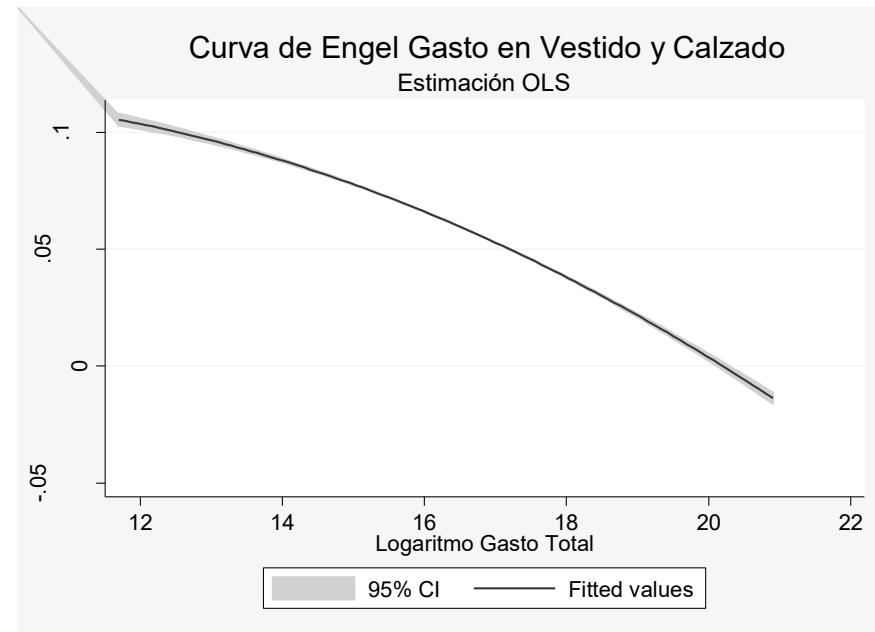

Figura 11. Curva de Engel: gasto vestido y calzado OLS

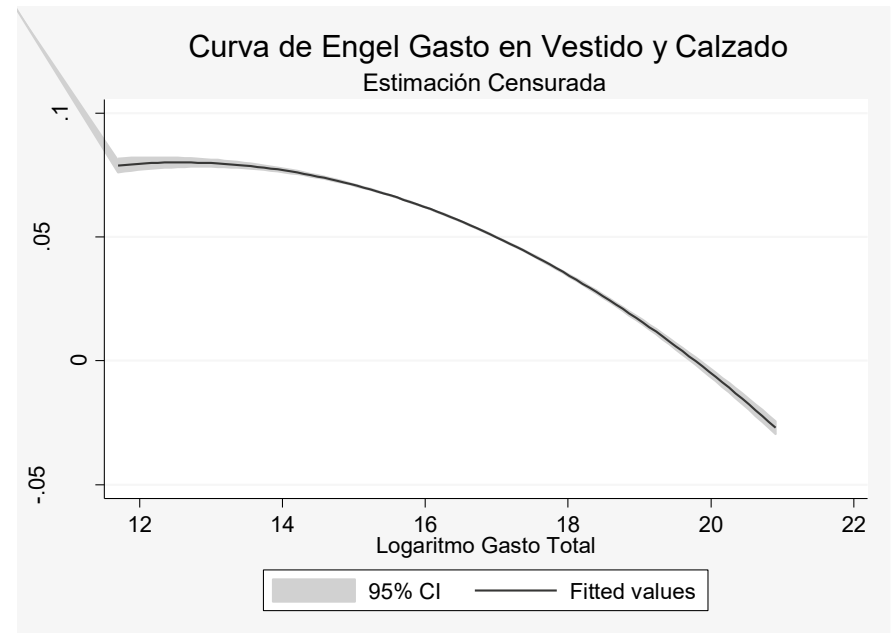

Figura 12. Curva de Engel: gasto en vestido y calzado censurada 


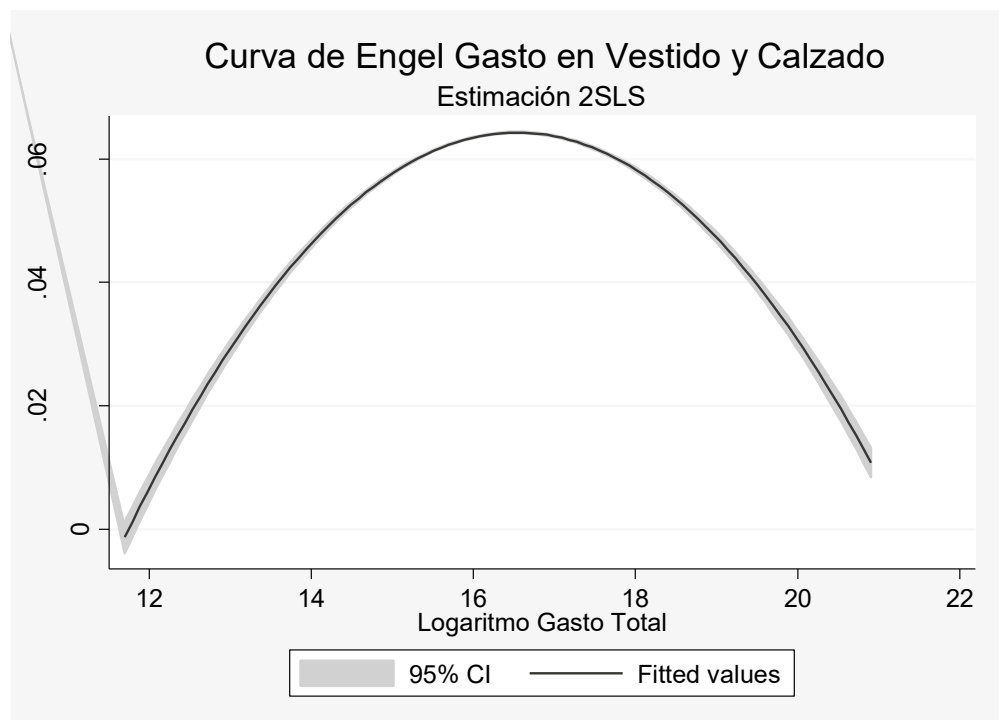

Figura 13. Curva de Engel: gasto en vestido 2SLS

Tabla 4. Estimación curva de Engel: gasto en vestido y calzado, 2012-2015

\begin{tabular}{cccc}
\hline Regresión & OLS & Tobit & $\begin{array}{c}\text { Variables } \\
\text { instrumentales }\end{array}$ \\
\hline Variable dependiente $(W v c)$ & Coeficiente & Coeficiente & Coeficiente \\
\hline N.o Personas por hogar & 0,0051 & 0,0052 & 0,0030 \\
& $(25,41)$ & $(26,81)$ & $(14,03)$ \\
\hline Sexo & 0,0023 & 0,0027 & $-0,0008$ \\
& $(4,15)$ & $(4,42)$ & $(1,38)$ \\
\hline Ln(edad) & $-0,0250$ & $-0,0286$ & $-0,0232$ \\
& $(26,60)$ & $(29,48)$ & $(24,10)$ \\
\hline Ln(GT*GT) & $-0,0005$ & $-0,0011$ & $-0,0011$ \\
& $(0,99)$ & $(3,12)$ & $(1,37)$ \\
\hline Ln(GT) & $-0,0010$ & 0,0207 & 0,0436 \\
& $(0,06)$ & $(1,75)$ & $(1,63)$ \\
\hline Estrato 2 & 0,0048 & 0,0044 & $-0,0012$ \\
& $(5,00)$ & $(4,28)$ & $(1,18)$ \\
\hline Estrato 3 & 0,0081 & 0,0072 & $-0,0077$ \\
& $(7,27)$ & $(6,44)$ & $(6,51)$ \\
\hline & & & \\
\hline
\end{tabular}




\section{Continuación Tabla 4}

\begin{tabular}{cccc}
\hline Estrato 4 & 0,0126 & 0,0101 & $-0,0144$ \\
& $(8,53)$ & $(6,94)$ & $(9,21)$ \\
\hline Estrato 5 & 0,0156 & 0,0115 & $-0,0196$ \\
& $(9,25)$ & $(6,99)$ & $(10,40)$ \\
\hline Estrato 6 & 0,0284 & 0,0265 & $-0,0190$ \\
& $(11,47)$ & $(12,43)$ & $(6,37)$ \\
\hline Constante & 0,2925 & 0,1069 & $-0,2624$ \\
& $(1,91)$ & $(1,10)$ & $(1,20)$ \\
\hline Número de observaciones & 47.326 & 47.326 & 47.326 \\
\hline Coeficiente de determinación & 0,0566 & $* * *$ & 0,0113 \\
\hline
\end{tabular}

Valor absoluto de la $t$-student entre paréntesis, al $5 \%$ de significancia. $\left(^{*}\right) \mathrm{Wvc}$ : asto en vestido y calzado / Gasto total

Varias observaciones acerca del impacto de las variables explicativas sobre el gasto en alimentación, bebida y tabaco, y vestido y calzado. El número de personas en el hogar implica incrementos significativamente estadísticos sobre el gasto en alimentación, no así en el de bebida y tabaco, y moderado (respecto al de alimentación) sobre el gasto en vestido y zapatos. El que el jefe de hogar sea hombre y su edad no tienen un efecto discernible sobre el gasto en alguno de los rubros, es decir, el efecto es el mismo, positivo pero sin grandes diferencias. El coeficiente asociado al gasto total no se interpreta, realmente es la curva de Engel, por tanto las interpretaciones se hicieron con base en las figuras presentadas. El estrato lo usamos para controlar por el ingreso y otras características menos observables, sin detrimento del gasto total como variable clave en la estimación de la media condicional; en las tres estimaciones, el efecto, en promedio, siempre es más pequeño respecto al estrato 1 , que es el de referencia mientras se mejora el estrato (los hogares se mueven de manera ascendente). Esto significa que los hogares más pobres son los que más restricciones presupuestales enfrentan, aunque son más sensibles al gasto en alimentación.

\section{CONCLUSIONES}

Según las estimaciones, estadísticamente se verifica el cumplimiento de la ley de Engel, la cual afirma que, a mayores ingresos, el porcentaje destinado a alimentación disminuye. Para los hogares de ingresos más bajos indagados en la encuesta, el gasto en alimentación se lleva una gran proporción del gasto; en promedio para las familias de ingresos bajos, el $35 \%$ del gasto del hogar se va en alimentación. Al tomar todas las familias, el gasto del hogar en alimentación es de aproximadamente el $43 \%$. 
Así mismo, los bienes alimentación, bebida y tabaco, y vestido y calzado, responden a la ley de Engel, y dependiendo del segmento de ingreso/gasto en el cual se ubiquen las familias, para algunas será un bien normal o inferior o de lujo. Esto se verifica al permitir que las regresiones controlen por más curvatura del gasto total y cuando se controla el sesgo debido a la censura en los diferentes rubros de gastos, así como cuando instrumentamos el gasto total con el ingreso de los hogares debido a la endogeneidad por simultaneidad.
Un punto importante es que se incluye más curvatura debido al diagnóstico preliminar llevado a cabo con una estimación no-parámetrica unidimensional. Se encontró también que el número de personas en el hogar tiene un efecto positivo en todos los rubros de gasto, aunque muy débil; el jefe de hogar hombre aumenta ligeramente el gasto en los diferentes rubros, y la edad del jefe de hogar no tiene efecto significativo sobre el gasto en bebida y tabaco, reduce el gasto en vestido y calzado, aunque aumenta el gasto en alimentación. 


\section{REFERENCIAS}

Baltagi, B. (2005). Econometric Analysis of Panel Data (3th ed.). Hoboken, NJ: John Wiley and Sons.

Banks, J. Blundell, R. \& Lewbel, A. (1997). Quadratic Engel Curves and Consumer Demand. The Review of Economics and Statistics, 79(4), 527-539.

Bhalotra, S. \& Attfield, C (1998) Intrahousehold Resources Allocation in Rural Pakistan: Semiparametric Analysis. Journal of Applied Econometrics, 13(5), 463-480.

Barrientos, J. (2009, ene.-jun.). Consumer Behaviour in Urban Colombia: The Case of Bogotá. Ensayo Sobre Politica Económica ESPE, (59).

Barrientos, J. (2011) Sobre la curva de Engel de la salud en Colombia. Una aproximación semiparamétrica. Lecturas de Economía, (74).

Blundell, R., Duncan, A. \& Pendakur, K. (1998). Semiparametric Estimation and Consumer Demand. Journal of Applied Econometrics, 13(5), 435-461.

Blundell, R., Browning, M. \& Crawford, I. (2003) Non-parametric Engel Curve and Revelead Preference. Econometrica, 71(1), 205-240.

Deaton, A. \& Muellbauer, J. (1980a). An almost Ideal Demand System. American Economic Review, 70(3), 312-26.

Deaton, A. \& Muellbauer, J. (1980b). Economics and Consumer Behavior. Cambridge: Cambridge University Press.

Leser, C. E. V. (1963). Form of Engel Functions. Econométrica, 31(4), 694-703.

Lyssiotou, P., Pashardes, P. \& Stengos, T. (2002). Age Effects on Consumer Demand: An Additive Partially Linear Regression Model. The Canadian Journal of Economics, 35(1), 153-165.

López, C. \& Gómez, H. (1976), El consumo entre las familias urbanas de Colombia. Coyuntura Económica, 6(2), 28. 
Moral-Arce, I., Rodríguez-Poo, J. \& Sperlich, S. (2013) Consumer Behavior Analysis for Luxury Goods. A Technical Note for Empirical Studies. Applied Economics Letters, 20, 358-363.

Nadaraya, E. A. (1964). On Estimating Regression. Theory Probability Applied, 10.

Newey, W. K. \& Powell, J. (2003). Instrumental Variables Estimation of Non-parametric Models. Econometrica, (71), 1565-1578.

Pindyck, R. S. \& Rubinfeld, D.L. (2016). Microeconomics. Boston: The Pearsons Series in Economics.

Ramírez, M., Cortés, D. \& Gallego, J. (2002, jul.-dic.). El gasto en salud de los hogares colombianos: un análisis descriptivo. Lecturas de Economía, (57).

Sperlich, S. (2005). A Note on Nonparametric Estimation with Constructed Variables and Generated Regressors. Social Science Research Network. http:// papers.ssrn.com/sol3/papers.cfm?abstract_id $=1010923$

Working, H. (1943). Statistical Laws of Family Expenditure. Journal of the American Statistical Association, 38, 4-56. 


\section{ANEXOS}

Tabla 5. Estadísticas descriptivas, 2015

\begin{tabular}{c|c|c|c|c|c}
\hline Variables & Observaciones & Media & $\begin{array}{c}\text { Desviación } \\
\text { Estándar }\end{array}$ & Mínimo & Máximo \\
\hline N. ${ }^{\circ}$ Personas/Hogar & 10620 & 3,3897 & 1,6098 & 1 & 13 \\
Sexo & 10620 & 0,5320 & 0,4990 & 0 & 1 \\
Edad & 10620 & 52,3986 & 15,6648 & 17 & 85 \\
Estrato 1 & 10620 & 0,1196 & 0,3245 & 0 & 1 \\
Estrato 2 & 10620 & 0,3341 & 0,4717 & 0 & 1 \\
Estrato 3 & 10620 & 0,2985 & 0,4576 & 0 & 1 \\
Estrato 4 & 10620 & 0,1166 & 0,3209 & 0 & 1 \\
Estrato 5 & 10620 & 0,0906 & 0,2870 & 0 & 1 \\
Estrato 6 & 10620 & 0,0407 & 0,1976 & 0 & 1 \\
\hline
\end{tabular}

Tabla 6. Estadísticas descriptivas, 2015

\begin{tabular}{c|c|c|c|c|c}
\hline Variables & Observaciones & Media & $\begin{array}{c}\text { Desviación } \\
\text { estándar }\end{array}$ & Mínimo & Máximo \\
\hline $\begin{array}{c}\text { Gasto } \\
\text { alimentación }\end{array}$ & 10620 & 0,4043 & 0,1930 & $-0,0025$ & 1 \\
$\begin{array}{c}\text { Gasto anual } \\
\text { alimentación }\end{array}$ & 10620 & 4.787 .915 & 2.908 .108 & -1.188 & 21.600 .000 \\
$\begin{array}{c}\text { Gasto mensual } \\
\text { total }\end{array}$ & 10620 & 1.242 .658 & 1.237 .337 & 25.000 & 15.000 .000 \\
$\begin{array}{c}\text { Gasto anual } \\
\text { total }\end{array}$ & 10620 & 14.900 .000 & 14.800 .000 & 300.000 & 180.000 .000 \\
$\begin{array}{c}\text { Ingreso anual } \\
\text { total }\end{array}$ & 10620 & 11.400 .000 & 8.367 .513 & 240.000 & 112.000 .000 \\
\hline
\end{tabular}


Tabla 7. Estimación Curva de Engel gasto en alimentación, 2015

\begin{tabular}{|c|c|c|c|}
\hline Regresión & OLS & Tobit & $\begin{array}{c}\text { Variables } \\
\text { instrumentales }\end{array}$ \\
\hline Variable dependiente (Wa) & Coeficiente & Coeficiente & Coeficiente \\
\hline \multirow[t]{2}{*}{ N. ${ }^{\circ}$ Personas/Hogar } & 0,0116 & 0,0115 & $-0,0024$ \\
\hline & $(9.79)$ & $(10.88)$ & $(1.86)$ \\
\hline \multirow[t]{2}{*}{ Sexo } & 0,0118 & 0,0113 & $-0,0063$ \\
\hline & $(3.60)$ & (3.43) & $(1.67)$ \\
\hline \multirow[t]{2}{*}{ Lnedad } & 0,0342 & 0,0337 & 0,0495 \\
\hline & $(6.97)$ & (6.69) & $(8.46)$ \\
\hline \multirow[t]{2}{*}{ Lngatot2 } & $-0,0265$ & $-0,0284$ & $-0,0896$ \\
\hline & $(9.02)$ & $(13.11)$ & $(9.26)$ \\
\hline \multirow[t]{2}{*}{ Lngatot } & 0,7423 & 0,8035 & 2,9589 \\
\hline & $(7.60)$ & $(11.36)$ & (9.31) \\
\hline \multirow[t]{2}{*}{ Estrato 2} & $-0,0287$ & $-0,0292$ & $-0,0635$ \\
\hline & (4.95) & $(5.30)$ & $(8.74)$ \\
\hline \multirow[t]{2}{*}{ Estrato 3} & $-0,0506$ & $-0,0515$ & $-0,1475$ \\
\hline & $(8.24)$ & $(8.80)$ & (18.69) \\
\hline \multirow[t]{2}{*}{ Estrato 4} & $-0,0713$ & $-0,0743$ & $-0,2046$ \\
\hline & $(8.77)$ & $(10.25)$ & $(21.09)$ \\
\hline \multirow[t]{2}{*}{ Estrato 5} & $-0,0697$ & $-0,0719$ & $-0,2274$ \\
\hline & $(7.71)$ & (8.94) & (20.64) \\
\hline \multirow[t]{2}{*}{ Estrato 6} & $-0,0551$ & $-0,0594$ & $-0,2452$ \\
\hline & $(5.00)$ & (5.33) & $(14.12)$ \\
\hline \multirow[t]{2}{*}{ Constante } & $-4,7631$ & $-5,2649$ & $-24,0330$ \\
\hline & $(5.89)$ & $(9.11)$ & $(9.21)$ \\
\hline Número de observaciones & 10620 & 10620 & 10620 \\
\hline Coeficiente de determinación & 0,2714 & $* * *$ & 0,0227 \\
\hline
\end{tabular}

